\title{
Resonant Decay of a Two State Atom Interacting with a Massless Non-Relativistic Quantised Scalar Field
}

\author{
Christopher King \\ Department of Mathematics, Northeastern University, Boston, MA 02115, USA
}

Received: 26 August 1993/ in revised form: 31 January 1994

\begin{abstract}
We consider a model Hamiltonian derived from the interaction of an atom with a non-relativistic massless quantized field. The model atom has two states, and the interaction is linear in the field operator. We do not make the rotating wave approximation and there is no infrared cutoff. We prove that the excited state of the atom with no photons present decays at an approximately exponential rate in accordance with the predictions of time dependent perturbation theory. The proof requires some analyticity and regularity assumptions on the interaction between atom and field. These imply in particular that the interaction goes to zero at least as fast as $k^{2}$, as $k \rightarrow 0$, where $k$ is the photon momentum.
\end{abstract}

\section{Introduction}

The subject of this paper is an important and widely studied topic in quantum mechanics, namely the interaction of an atom with a quantised field. The specific problem addressed here is to show that time dependent perturbation theory correctly describes the decay of an excited state of the atom over an intermediate time interval, for sufficiently weak coupling. For simplicity, the atom is assumed to have only two states, the ground state and an excited state. The object is to show that if the atom is in its excited state at time zero with no photons present, then the probability that it will remain in that state at future times decays at an approximately exponential rate. We prove this result with some analyticity assumptions on the interaction between atom and field.

The literature on this subject is huge; a partial list is [CMR, Da, Di, DE, EY, $\mathrm{F}$, Froh, $\mathrm{He}, \mathrm{Hu}, \mathrm{Ki}, \mathrm{OY}, \mathrm{Sk}, \mathrm{St}]$. For our purposes the most relevent results are in [OY], where the method of dilatation analyticity is applied to the full model of a one electron atom interacting with a massive quantised field (with an ultraviolet cutoff). It is shown there that the excited state becomes a resonance in the sense described in [AC, BC, Hu, S1, S2]. As shown by Hunziker [Hu], this then implies approximate exponential decay in the manner predicted by time dependent perturbation theory. In particular suppose that $\psi_{0}$ is the eigenstate of the unperturbed problem which is believed to become a resonance. If $H(\beta)$ is the interacting Hamiltonian, where $\beta$ is 
the coupling constant, then Hunziker shows that there is a positive number $a$ such that for all $t>0$, and $\beta$ sufficiently small,

$$
\left|\left(\psi_{0}, e^{-i t H(\beta)} \psi_{0}\right)\right|=e^{-\beta^{2} a t}+O\left(\beta^{2}\right),
$$

where the remainder $O\left(\beta^{2}\right)$ is uniform in $t$. This estimate allows an interpretation of both "decay rate" and "intermediate times," ideas which arise from time dependent perturbation theory. We shall prove that this result holds for our model also. We note that E.B. Davies [Da] has proved a result similar to (1.1) for this model, but without the estimate $O\left(\beta^{2}\right)$ for the remainder term.

The method used in [OY] requires a massive photon. This is a technical condition needed to apply the usual method of dilatation analyticity. On the other hand, the "infrared problem" in QED associated with the massless photon has a long history [B1, BN, Froh, FrMS], and arises in many different areas. The question addressed in this paper is one aspect of the infrared problem.

The full state space of our model is $C^{2} \otimes \mathscr{F}$, where $\mathscr{F}$ is the symmetric Fock space over $L^{2}(R)$. The excited state of the atom is $\left(\begin{array}{l}1 \\ 0\end{array}\right)$, and the ground state is $\left(\begin{array}{l}0 \\ 1\end{array}\right)$. With respect to this choice of basis, the Hamiltonian is

$$
\begin{aligned}
H= & \left(\begin{array}{cc}
E_{0} & 0 \\
0 & 0
\end{array}\right) \otimes 1+1 \otimes \int|k| a^{\dagger}(k) a(k) d k \\
& +\beta\left(\begin{array}{ll}
0 & 1 \\
1 & 0
\end{array}\right) \otimes \int\left(f(k) a^{\dagger}(k)+\overline{f(k)} a(k)\right) d k .
\end{aligned}
$$

Here $\left[a(k), a^{\dagger}\left(k^{\prime}\right)\right]=\delta\left(k-k^{\prime}\right)$ represents the scalar Bose field. Also $E_{0}>0$ is the gap between the ground state and excited state of the atom. The Hamiltonian (1.2) is identical to the spin-boson Hamiltonian which has been extensively studied as a model for tunneling [L-Z, SD]. To the best of our knowledge the result proved in this paper has not been derived in that context. Hamiltonians related to (1.2) have also been studied in the polaron problem [Froh, G, N2]. We shall give a more precise and detailed description of (1.2) in Sect. 2.

We tackle the problem using a hybrid of other methods. We adopt a strategy outlined in [Ki], which allows the approximate exponential decay (1.1) to be deduced from certain regularity bounds on a matrix element of the resolvent of the Hamiltonian near the putative resonance. We obtain these estimates using dilatation analytic methods. However, we dispense with the usual goal of dilatation analyticity, which is to find an analytic continuation of a matrix element of the resolvent across the continuous spectrum. As a technical aid, we first prove the result using an upper bound $N$ on the number of photons in any state. We then let $N \rightarrow \infty$ to deduce the final result.

Our method requires the assumption that the photon wave function $f(k)$ in (1.2) is dilatation analytic, and also that $|k|^{p} f(k)$ is square integrable, for $-3 / 2 \leqq p \leqq 1 / 2$. The precise conditions are stated in Definition 4.1. The conditions (ii) that $|k|^{-3 / 2} f(k)$ be square integrable and (iii) that $f^{\prime \prime}(k)$ be uniformly bounded imply in particular that $f(k)$ approaches zero at least as fast as $k^{2}$, when $k \rightarrow 0$. These rather strong conditions are needed in this approach to control the infrared problem. Removing these conditions would be an interesting extension of our results. 
The main difficulty is caused by the massless photon. Let $H_{\theta}(0)$ be the analytic dilatation of the free Hamiltonian $H(0)$ (this terminology is borrowed from [S1] and [OY], and will be explained in Sect. 4); from (1.2) we have

$$
H_{\theta}(0)=\left(\begin{array}{cc}
E_{0} & 0 \\
0 & 0
\end{array}\right) \otimes 1+1 \otimes \int e^{-\theta}|k| a^{\dagger}(k) a(k) d k
$$

The usual strategy at this point is to use standard Rayleigh-Schrödinger perturbation theory to introduce the perturbation in (1.3). However the massless photon means that the eigenvalue $E_{0}$ of (1.3) is not isolated from the continuous spectrum, so this does not work. The resolution of this problem is contained in Proposition 4.5 , which is the technical heart of the paper. Roughly speaking, it states that in each $n$-particle sector of the state space, the singularities of the analytically dilated resolvent are pulled below the real axis at low energies by the interaction with the $(n+1)$-particle sector. This interaction separates into a direct part and an exchange part. The direct term is simple to evaluate and produces the desired shift; the exchange term is much more complicated. However, the exchange term is short range and so does not contribute to the low energy behavior of the resolvent. This rough idea lies behind the results proved in this paper. This result is then used to prove that a partially resummed perturbation series for the full resolvent is convergent.

The paper is organised as follows. Section 2 contains the definition of the model and introduces the notation used throughout the paper. Section 3 states the main result in Theorem 3.1, and derives it from Theorem 3.2. This latter result concerns the regularity of a certain resolvent. In Sect. 4, this regularity result is derived from a resummed perturbation series, assuming some bounds on the "renormalised propagators" for the photon. These bounds are then derived in Sect. 5, where the infrared problem is met and overcome. Section 6 proves that the infinite photon number limit exists.

\section{Definition of the Model}

Our model is a caricature of the interaction between an atom and a non-relativistic massless quantised field. The essential features are that the atom has two states only, and that the interaction is linear in the field. The interaction is therefore the sum of a creation part $C(f)$ and an annihilation part $A(f)=C(f)^{*}$. The operator $C(f)$ creates a photon with wave function $f \in L^{2}(R)$. For simplicity we use a onedimensional field. The wave function $f$ is the only remnant in this model of the wave functions of the ground state and excited state of the atom. We will make further assumptions on $f$ shortly.

The interactions $C(f)$ and $A(f)$ are multiplied by operators which switch the two states of the atom. This is in accord with the usual semiclassical description of photon emission and absorption. For simplicity we ignore interactions which change the photon number but leave the atomic state unchanged.

We note that our model includes interaction terms corresponding to photon emission accompanied by atomic transition from ground state to excited state, and its conjugate. Therefore our model goes beyond the rotating wave approximation, which has been extensively analysed [see for example the review article [St] by Stenholm].

As mentioned in the introduction, we introduce a cutoff $N$ on the number of photons. Our estimates are uniform in $N$, and at the end we let $N \rightarrow \infty$ to obtain the 
result for the full model. Our coupling constant is denoted $\beta$. Rather than deriving the Hamiltonian $H(\beta)$ from the full non-relativistic model, we give a self-contained description below, which avoids introducing extraneous spaces and operators.

Due to our simplified choice of interaction, the full state space is a sum of two non-interacting parts. One consists of states which have an even number of photons with the atom in its excited state, and an odd number of photons with the atom in its ground state. The other part has the "even" and "odd" switched. We shall be interested only in the former part, which can be identified with the complex Hilbert space

$$
\mathscr{H}=\mathscr{F}_{0} \oplus \mathscr{F}_{1} \oplus \cdots \oplus \mathscr{F}_{N} .
$$

Here $\mathscr{F}_{0}=C, \mathscr{F}_{1}=L^{2}(R)$, and $\mathscr{F}_{n}$ is the symmetric subspace of $L^{2}(R)^{\otimes n}$, corresponding to $n$-photon states. Specifically, let $S_{n}$ be the symmetric group on $n$ letters. Then $F\left(k_{1}, \ldots, k_{n}\right)$ is in $\mathscr{F}_{n}$ if for all $\sigma \in S_{n}$,

$$
F\left(k_{1}, \ldots, k_{n}\right)=F\left(k_{\sigma(1)}, \ldots, k_{\sigma(n)}\right) .
$$

The free photon Hamiltonian is diagonal with respect to the decomposition (2.1), and on $\mathscr{F}_{n}$ is given by the operator $g_{n}$, defined as follows:

$$
\left(g_{n} F\right)\left(k_{1}, \ldots, k_{n}\right)=\left(\left|k_{1}\right|+\cdots+\left|k_{n}\right|\right) F\left(k_{1}, \ldots, k_{n}\right) .
$$

We define $g_{0}=0$. Let $E_{0}>0$ denote the energy difference between the excited state and the ground state of the atom. Then the non-interacting Hamiltonian $H(0)$ is also diagonal with respect to the decomposition (2.1), and is given on $\mathscr{F}_{n}$ by

$$
\left.H(0)\right|_{\mathscr{F}_{n}}:=G_{n}=\left\{\begin{array}{ll}
g_{n}+E_{0} & n \text { even } \\
g_{n} & n \text { odd }
\end{array} .\right.
$$

Let $P_{n}: \mathscr{H} \rightarrow \mathscr{F}_{n}$ be the orthogonal projection for $0 \leqq n \leqq N$, and let $P_{n}^{*}$ denote its adjoint. The creation operator $C(f): \mathscr{H} \rightarrow \mathscr{H}$ is defined as follows; for all $0 \leqq n \leqq N-1$, and $F \in \mathscr{F}_{n}$,

$$
\begin{aligned}
P_{m} C(f) P_{n}^{*} F & =0 \quad \text { if } m \neq n+1, \\
\left(P_{n+1} C(f) P_{n}^{*} F\right)\left(k_{1}, \ldots, k_{n+1}\right) & =\frac{1}{\sqrt{n+1}}\left\{\sum_{j=1}^{n+1} f\left(k_{j}\right) F\left(k_{1}, \ldots, \hat{k}_{j}, \ldots, k_{n+1}\right)\right\} .
\end{aligned}
$$

We use $\hat{k}_{j}$ to indicate that this entry is missing. Similarly the annihilation operator $A(f)$ is given by its action on $\mathscr{F}_{n}$ as follows:

$$
\begin{aligned}
P_{m} A(f) P_{n}^{*} F & =0 \quad \text { if } m \neq n-1, \quad \text { or if } n=0, \\
\left(P_{n-1} A(f) P_{n}^{*} F\right)\left(k_{1}, \ldots, k_{n-1}\right) & =\sqrt{n} \int \overline{f\left(k_{n}\right)} F\left(k_{1}, \ldots, k_{n}\right) d k_{n} .
\end{aligned}
$$

Note that $C(f)$ is linear in $f$, and $A(f)$ is anti-linear in $f$. To define the interacting Hamiltonian $H(\beta)$, it is convenient to introduce the subspaces

$$
\mathscr{G}_{n}=\mathscr{F}_{n} \oplus \mathscr{F}_{n+1} \oplus \cdots \oplus \mathscr{F}_{N} .
$$

Let $Q_{n}: \mathscr{G}_{n} \rightarrow \mathscr{F}_{n}$ be the orthogonal projection, and let $Q_{n}^{*}$ denote its adjoint. We define for $0 \leqq n \leqq N-1$,

$$
\begin{aligned}
& C_{n+1}=Q_{n+1}^{*} P_{n+1} C(f) P_{n}^{*}: \mathscr{F}_{n} \rightarrow \mathscr{G}_{n+1}, \\
& A_{n+1}=P_{n} A(f) P_{n+1}^{*} Q_{n+1}: \mathscr{G}_{n+1} \rightarrow \mathscr{F}_{n} .
\end{aligned}
$$


The interacting Hamiltonian $H(\beta)$ is defined recursively as follows. For each $0 \leqq n \leqq N$, we let $h_{n}$ denote the restriction of $H(\beta)$ to states with $n$ or more photons. So $h_{n}: \mathscr{G}_{n} \rightarrow \mathscr{G}_{n}$, with $h_{0}=H(\beta)$ and $h_{N}=G_{N}$. Write $\mathscr{H}=\mathscr{G}_{0}=$ $\mathscr{F}_{0} \oplus \mathscr{G}_{1}$; then with respect to this decomposition,

$$
H(\beta)=\left(\begin{array}{cc}
E_{0} & \beta A_{1} \\
\beta C_{1} & h_{1}
\end{array}\right)
$$

For each $1 \leqq n \leqq N-1$, write $\mathscr{G}_{n}=\mathscr{F}_{n} \oplus \mathscr{G}_{n+1}$; then with respect to this decomposition,

$$
h_{n}=\left(\begin{array}{cc}
G_{n} & \beta A_{n+1} \\
\beta C_{n+1} & h_{n+1}
\end{array}\right) .
$$

The reason for writing the operator in this way is that it allows a simple recursive evaluation of the resolvent of $H(\beta)$. Our principal object of study is the amplitude $\left(\psi_{0}, e^{-i t H(\beta)} \psi_{0}\right)$ described in the introduction, which in this notation is

$$
A(t):=P_{0} e^{-i t H(\beta)} P_{0}^{*} \text {. }
$$

For $\operatorname{Im}(z) \neq 0$, we define

$$
R(z)=P_{0}(H(\beta)-z)^{-1} P_{0}^{*} .
$$

The spectral theorem implies that

$$
A(t)=\lim _{\varepsilon \rightarrow 0+} \frac{1}{2 \pi i} \int_{-\infty}^{\infty} e^{-i \lambda t}[R(\lambda+i \varepsilon)-R(\lambda-i \varepsilon)] d \lambda .
$$

So we need an expression for $P_{0}(H(\beta)-z)^{-1} P_{0}^{*}$, the projection of the resolvent onto the excited state of the atom with no photons present. For $0 \leqq n \leqq N-1$, and $\operatorname{Im}(z) \neq 0$, define

$$
\begin{gathered}
M_{n}(z): \mathscr{F}_{n} \rightarrow \mathscr{F}_{n}, \\
M_{n}(z)=A_{n+1}\left(h_{n+1}-z\right)^{-1} C_{n+1} .
\end{gathered}
$$

Then by resumming the Neumann series for (2.9) (see [Ki]) we see that for $\operatorname{Im}(z) \neq 0$,

$$
P_{0}(H(\beta)-z)^{-1} P_{0}^{*}=\left(E_{0}-z-\beta^{2} M_{0}(z)\right)^{-1} .
$$

In a similar way, it follows from (2.10) that for all $0 \leqq n \leqq N-1$,

$$
M_{n}(z)=A_{n+1} Q_{n+1}^{*}\left(G_{n+1}-z-\beta^{2} M_{n+1}(z)\right)^{-1} Q_{n+1} C_{n+1} .
$$

Also $M_{N}(z)=0$. Using (2.15) and $M_{0}(\bar{z})=\overline{M_{0}(z)}$ (for $\operatorname{Im} z \neq 0$ ), (2.13) can be rewritten as

$$
A(t)=\lim _{\varepsilon \rightarrow 0+} \frac{1}{\pi} \int_{-\infty}^{\infty} e^{-i \lambda t} \operatorname{Im}\left[E_{0}-\lambda-i \varepsilon-\beta^{2} M_{0}(\lambda+i \varepsilon)\right]^{-1} d \lambda .
$$

Our result, which is stated in the next section, requires some additional assumptions on the interaction function $f$. We assume that $f(0)=0$ and that $f\left(E_{0}\right) \neq 0$. We also assume that $f$ is a dilatation analytic vector in the sense of Nelson [N1] and Simon [S1], and that its analytic continuation satisfies some regularity conditions. This property is defined in Sect. 4, where the precise assumptions on $f$ are also stated. 


\section{Results}

Our main result concerns the behavior of the amplitude $A(t)$ given in (2.11), for $t>0$ and for $\beta$ sufficiently small.

Theorem 3.1. Assume $f$ satisfies condition $(R)$ given in Definition 4.1. Then there are numbers $\beta_{0}>0, a>0$ and $E_{1}$, independent of $N$, such that for $|\beta| \leqq \beta_{0}$ and all $t>0$,

a)

$$
A(t)=e^{-i E_{0} t-i \beta^{2} E_{1} t} e^{-a \beta^{2} t}+O\left(\beta^{2}\right),
$$

b)

$$
\lim _{N \rightarrow \infty} A(t) \text { exists . }
$$

Remark. The result (3.1) has been derived for other models which display resonant behavior (see $[\mathrm{Hu}]$ and [Ki] for examples). It implies approximate exponential decay of the amplitude $A(t)$, whose square $|A(t)|^{2}$ is the probability that the atom is in its excited state at time $t$ with no photons present, given that it was excited at time zero with no photons present. The remainder $O\left(\beta^{2}\right)$ in (3.1) is uniform in $t$. In fact the left side of (3.1) goes to zero as $t \rightarrow \infty$, but at a rate slower than exponential [S2].

Unless otherwise stated, all estimates and constants from here on are independent of $\beta$ and $N$. The proof of Theorem 3.1 uses the representation (2.17) and the following result.

Theorem 3.2. There are positive numbers $\beta_{1}, \alpha$ such that for all $|\beta| \leqq \beta_{1}$,

i) $M_{0}(z)$ and its first three derivatives are bounded uniformly in $\beta$ and $z$, for all $|\beta| \leqq \beta_{1}$, and for all $z$ satisfying $\operatorname{Im} z>0,\left|\operatorname{Re} z-E_{0}\right| \leqq \alpha$;

ii) $\lim _{\varepsilon \rightarrow 0+} \operatorname{Im} M_{0}\left(E_{0}+i \varepsilon\right)=a>0$.

We will use Theorem 3.2 to prove Theorem 3.1(a). The proof of Theorem 3.2, which uses dilatation analyticity, is given in Sects. 4 and 5 . Theorem 3.1(b) is proved in Sect. 6. First we have the following result.

Lemma 3.3. Let $h(x, y)$ be defined for $y>0$ and $a \leqq x \leqq b$. Suppose that for some integer $k \geqq 1$, and some positive numbers $c, M$,

$$
\sup \left\{\left|\partial^{\alpha} h(x, y)\right|: a \leqq x \leqq b, 0<y \leqq c, \quad 0 \leqq|\alpha| \leqq k\right\} \leqq M .
$$

Then for each $x$ in $[a, b]$, there exists $g(x)=\lim _{y \rightarrow 0+} h(x, y)$. Furthermore $g$ is continuous in $[a, b]$, and has at least $k-1$ continuous derivatives in $(a, b)$.

Proof. Let $x \in[a, b]$, and let $\left\{y_{n}\right\}$ be any decreasing sequence converging to zero. For all $n, m$ sufficiently large,

$$
\begin{aligned}
\left|h\left(x, y_{n}\right)-h\left(x, y_{m}\right)\right| & \leqq\left|y_{n}-y_{m}\right| \sup \left|\frac{\partial h}{\partial y}\right| \\
& \leqq M\left|y_{n}-y_{m}\right|
\end{aligned}
$$

Therefore $\left\{h\left(x, y_{n}\right)\right\}$ is a Cauchy sequence, and we define

$$
g(x)=\lim _{n \rightarrow \infty} h\left(x, y_{n}\right) \text {. }
$$


The bound (3.2) implies uniqueness of the limit (3.3). Continuity of $g$ follows from a standard " $\frac{\varepsilon}{3}$ " argument and the uniform continuity of $h$.

Suppose now that $k \geqq 2$. Repeating the above argument leads to the continuous function

$$
G(x)=\lim _{y \rightarrow 0+} \frac{\partial h}{\partial x}(x, y) .
$$

For each $0<y \leqq c, h(x, y)-h(a, y)=\int_{a}^{x} \frac{\partial h}{\partial x}(s, y) d s$. Since $\frac{\partial h}{\partial x}$ is uniformly bounded, we may apply the Dominated Convergence Theorem to conclude that for all $x$ in $[a, b]$,

$$
g(x)-g(a)=\int_{a}^{x} G(s) d s .
$$

This implies that $G(x)=g^{\prime}(x)$ and hence that $g$ has one continuous derivative in $(a, b)$. Repeating the argument with higher derivatives of $h$ gives the stated result. QED

Applying Lemma 3.3 to $M_{0}(z)$, and using Theorem 3.2(i), we conclude that for $|\beta| \leqq \beta_{1}$, and real $\lambda$ satisfying $\left|\lambda-E_{0}\right| \leqq \alpha, M_{0}(\lambda):=\lim _{\varepsilon \rightarrow 0+} M_{0}(\lambda+i \varepsilon)$ exists and has at least two continuous derivatives in the interval $\left(E_{0}-\alpha, E_{0}+\alpha\right)$. Let

$$
K=\sup \left\{\left|M_{0}(z)\right|,\left|M_{0}^{\prime}(z)\right|,\left|M_{0}^{\prime \prime}(z)\right|: \operatorname{Im} z>0,\left|\operatorname{Re} z-E_{0}\right| \leqq \alpha\right\} .
$$

By Theorem 3.2 (i), $K$ is finite. Recall that for $\operatorname{Im} z \neq 0$, we have

$$
R(z)=P_{0}(H(\beta)-z)^{-1} P_{0}^{*}=\left(E_{0}-z-\beta^{2} M_{0}(z)\right)^{-1} .
$$

Lemma 3.4. There is $O<\alpha_{1} \leqq \alpha, 0<\beta_{2} \leqq \beta_{1}$ and $K_{1}>0$, such that for all $|\beta| \leqq \beta_{2}$ and all $z$ satisfying $\operatorname{Im} z>0,\left|\operatorname{Re} z-E_{0}\right| \leqq \alpha_{1}$,

$$
|R(z)| \leqq K_{1} \min \left\{\left|z-E_{0}\right|^{-1}, \beta^{-2}\right\} .
$$

Proof. If $\left|z-E_{0}\right| \geqq 2 \beta^{2} K$, then

$$
\left|R(z)^{-1}\right| \geqq\left|z-E_{0}\right|-\beta^{2} K \geqq \frac{1}{2}\left|z-E_{0}\right| \geqq \beta^{2} K .
$$

If $\left|z-E_{0}\right|<2 \beta^{2} K$, then Theorem 3.2 (i), (ii) imply

$$
-\operatorname{Im} R(z)^{-1}>\beta^{2} \operatorname{Im} M_{0}(z) \geqq \beta^{2}\left(a-\left|z-E_{0}\right| K\right) .
$$

Let $\alpha_{1}=\min \{\alpha, a / 2 K\}$, and $\beta_{2}^{2}=\min \left\{\beta_{1}^{2}, \alpha_{1} / 2 K\right\}$. Then for $\left|z-E_{0}\right| \leqq \alpha_{1}$ and $|\beta| \leqq \beta_{2}$,

$$
\left|R(z)^{-1}\right| \geqq\left|\operatorname{Im} R(z)^{-1}\right| \geqq \frac{a}{2} \beta^{2}>\frac{a}{4 K}\left|z-E_{0}\right| .
$$

Let $K_{1}^{-1}=\min \{1 / 2, K, a / 2, a / 4 K\}$; then $R(z)$ satisfies (3.4). QED

For $0<|\beta| \leqq \beta_{2}$, and $\left|\lambda-E_{0}\right| \leqq \alpha_{1}$, we define

$$
R(\lambda):=\lim _{\varepsilon \rightarrow 0+} R(\lambda+i \varepsilon)=\left(E_{0}-\lambda-\beta^{2} M_{0}(\lambda)\right)^{-1} .
$$

Let us write, for $\left|\lambda-E_{0}\right| \leqq \alpha_{1}$, 


$$
M_{0}(\lambda)=M_{0}\left(E_{0}\right)+\left(\lambda-E_{0}\right) M_{0}^{\prime}\left(E_{0}\right)+S(\lambda, \beta)
$$

where the remainder satisfies the bound

$$
|S(\lambda, \beta)| \leqq \frac{K}{2}\left|\lambda-E_{0}\right|^{2}
$$

Let $\beta_{3}^{2}=\min \left\{\beta_{2}^{2}, 1 / 2 K, a / 4 K^{2}\right\}$, and let $\xi=\left(1+\beta^{2} M_{0}^{\prime}\left(E_{0}\right)\right)^{-1}$. Then for $|\beta| \leqq \beta_{3}$ and $\left|\lambda-E_{0}\right| \leqq \alpha_{1}$, we have $|\xi| \leqq 2$ and

$$
R(\lambda)=-\xi\left(\lambda-E_{0}+\beta^{2} \xi M_{0}\left(E_{0}\right)+\beta^{2} \xi S(\lambda, \beta)\right)^{-1} .
$$

Furthermore, $\operatorname{Im} \xi M_{0}\left(E_{0}\right)>a / 2$ for all $|\beta| \leqq \beta_{3}$. So for $|\beta| \leqq \beta_{3},\left|\lambda-E_{0}\right| \leqq \alpha_{1}$ we define

$$
R_{0}(\lambda)=-\xi\left(\lambda-E_{0}+\beta^{2} \xi M_{0}\left(E_{0}\right)\right)^{-1}
$$

Then we have

$$
R(\lambda)-R_{0}(\lambda)=\beta^{2} S(\lambda, \beta) R(\lambda) R_{0}(\lambda)
$$

Lemma 3.5. For $|\beta| \leqq \beta_{3}$, there is a positive number $K_{2}$ such that

$$
\left|R_{0}(\lambda)\right| \leqq \frac{K_{2}}{\left|\lambda-E_{0}\right|}
$$

Proof. Let $r=\operatorname{Re}\left(\xi M_{0}\left(E_{0}\right)\right)$, and $s=\operatorname{Im}\left(\xi M_{0}\left(E_{0}\right)\right)$. Then $|r| \leqq 2 K$ and $s>a / 2$, so

$$
\begin{aligned}
\left|\lambda-E_{0}\right| & =\left|\lambda-E_{0}+\beta^{2} r-\beta^{2} r\right| \\
& \leqq\left|\lambda-E_{0}+\beta^{2} r\right|+\beta^{2}|r| \\
& =\left|\lambda-E_{0}+\beta^{2} r\right|+\beta^{2} \frac{|r|}{s} s \\
& \leqq\left|\lambda-E_{0}+\beta^{2} r\right|+\beta^{2} \frac{2}{a} 2 K s \\
& \leqq \sqrt{2} \max \left\{1, \frac{4 K}{a}\right\}\left|\lambda-E_{0}+\beta^{2} r+i \beta^{2} s\right|
\end{aligned}
$$

Combined with the bound $|\xi| \leqq 2$, this gives (3.10) with $K_{2}=2 \sqrt{2} \max \{1,4 K / a\}$. QED

Lemma 3.4 implies that for $|\beta| \leqq \beta_{2}$,

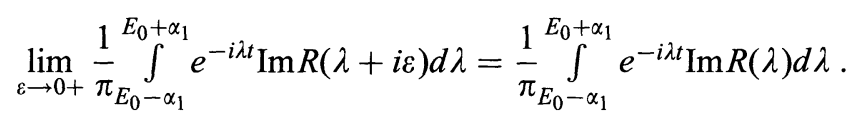

From (3.6), (3.9) and Lemmas 3.4 and 3.5, we deduce that for $|\beta| \leqq \beta_{3}$ and $\left|\lambda-E_{0}\right| \leqq \alpha_{1}$,

$$
\left|R(\lambda)-R_{0}(\lambda)\right| \leqq \beta^{2} \frac{K}{2} K_{1} K_{2}
$$


Therefore for $|\beta| \leqq \beta_{3}$,

$$
\left|\frac{1}{\pi} \int_{E_{0}-\alpha_{1}}^{E_{0}+\alpha_{1}} e^{-i \lambda t} \operatorname{Im}\left(R(\lambda)-R_{0}(\lambda)\right) d \lambda\right| \leqq \frac{2}{\pi} \alpha_{1} \beta^{2} \frac{K}{2} K_{1} K_{2} .
$$

Furthermore it is straightforward to show using a contour integral that there are positive numbers $K_{3}, K_{4}$, independent of $\beta$ and $t$, such that for all $t \geqq 0$ and all $|\beta| \leqq \beta_{3}$

$$
\begin{gathered}
\mid \frac{1}{\pi} \int_{E_{0}-\alpha_{1}}^{E_{0}+\alpha_{1}} e^{-i \lambda t} \operatorname{Im}\left(\lambda-E_{0}+\beta^{2} \xi M_{0}\left(E_{0}\right)^{-1} d \lambda+e^{-i\left(E_{0}-\beta^{2} r\right) t} e^{-\beta^{2} s t} \mid \leqq \beta^{2} K_{3}\right. \\
\left|\frac{1}{\pi} \int_{E_{0}-\alpha_{1}}^{E_{0}+\alpha_{1}} e^{-i \lambda t} \operatorname{Re}\left(\lambda-E_{0}+\beta^{2} \xi M_{0}\left(E_{0}\right)\right)^{-1} d \lambda\right| \leqq K_{4},
\end{gathered}
$$

where $r=\operatorname{Re}\left(\xi M_{0}\left(E_{0}\right)\right)$ and $s=\operatorname{Im}\left(\xi M_{0}\left(E_{0}\right)\right)$. Since $|\xi-1| \leqq 2 \beta^{2} K$, this and (3.13) imply that

$$
\frac{1}{\pi} \int_{E_{0}-\alpha_{1}}^{E_{0}+\alpha_{1}} e^{-i \lambda t} \operatorname{Im} R(\lambda) d \lambda=e^{-i\left(E_{0}-\beta^{2} r\right) t} e^{-\beta^{2} s t}+O\left(\beta^{2}\right) .
$$

Now $A(0)=1$, and $\operatorname{Im} R(\lambda+i \varepsilon) \geqq 0$ for all $\lambda$, and all $\varepsilon>0$. Therefore

$$
\begin{aligned}
\left|A(t)-e^{-i\left(E_{0}-\beta^{2} r\right) t} e^{-\beta^{2} s t}\right| & \leqq \lim _{\varepsilon \rightarrow 0+} \frac{1}{\pi} \int_{\left|\lambda-E_{0}\right|>\alpha_{1}} \operatorname{Im} R(\lambda+i \varepsilon) d \lambda+O\left(\beta^{2}\right) \\
& =A(0)-\lim _{\varepsilon \rightarrow 0+} \frac{1}{\pi} \int_{E_{0}-\alpha_{1}}^{E_{0}+\alpha_{1}} \operatorname{Im} R(\lambda+i \varepsilon) d \lambda+O\left(\beta^{2}\right) \\
& =O\left(\beta^{2}\right) .
\end{aligned}
$$

Finally, write $E_{1}=\operatorname{Re}\left(M_{0}\left(E_{0}\right)\right)$. Then $\left|r-E_{1}\right| \leqq 2 \beta^{2} K^{2}$, and $|s-a| \leqq 2 \beta^{2} K^{2}$, and so for all $t>0$,

$$
\begin{aligned}
\left|e^{i \beta^{2} r t-\beta^{2} s t}-e^{\imath \beta^{2} E_{1} t-\beta^{2} a t}\right| & \leqq e^{-\beta^{2} a t}\left|1-e^{i \beta^{2}\left(r-E_{1}\right) t-\beta^{2}(s-a) t}\right| \\
& \leqq 4 \beta^{4} t K^{2} e^{-\beta^{2} a t / 2} \\
& \leqq \frac{8 K^{2}}{a} \beta^{2}
\end{aligned}
$$

This proves Theorem 3.1(a) with $\beta_{0}=\beta_{3}$.

\section{Dilatation Analyticity and Theorem 3.2}

As mentioned in the introduction, we will use the method of dilatation analyticity to prove Theorem 3.2. The description below follows [N1, S1 and OY]. For $\theta$ real, we define the unitary dilation operator $U(\theta)$ on $L^{2}(R, d k)$ by

$$
U(\theta) \psi(k)=e^{-\frac{\theta}{2}} \psi\left(e^{-\theta} k\right) .
$$


Formally, $U(\theta)=e^{-\theta D}$, where $D=\frac{1}{2}\left(k \frac{d}{d k}+\frac{d}{d k} k\right)$ is the generator of dilatations. Let $N_{b}$ be the subspace of functions $\psi$ for which the series $\sum_{n=0}^{\infty} \frac{z^{n}}{n !} D^{n} \psi$ has radius of convergence at least $b$. We call $\psi$ a dilatation analytic vector if $\psi \in N_{b}$ for some $b>0$. If $\psi \in N_{b}$, then $U(\theta) \psi$ has an analytic continuation $\psi_{\theta}$ to the disk $|\theta| \leqq b$, given by

$$
\psi_{\theta}:=\sum_{n=0}^{\infty} \frac{(-\theta)^{n}}{n !} D^{n} \psi
$$

Although we will not need the result, we note that if $\psi \in N_{b}$, then $U(\theta) \psi$ can also be continued to the strip $|\operatorname{Im} \theta| \leqq b$. As discussed in Sect. 2, we will assume that the photon wave function $f$ which appears in the Hamiltonian $H(\beta)$ belongs to $N_{b}$ for some $b>0$. For later convenience, we also assume that $b<\pi / 8$. The following definition collects together the regularity properties of $f$ which we require for the results in this paper. Recall that $g_{1}$ defined in (2.3) is multiplication by $|k|$.

Definition 4.1. The function $f$ satisfies the regularity condition $(R)$ if the following holds:

i) for some $0<b<\pi / 8, f \in N_{b}$;

ii) $\left\|g_{1}^{p} f_{\theta}\right\|_{2}$ is uniformly bounded for all $|\theta| \leqq b$, and all $-\frac{3}{2} \leqq p \leqq \frac{1}{2}$;

iii) $|k|^{-1 / 2}\left|f_{\theta}(k)\right|,\left|f_{\theta}^{(n)}(k)\right|$ are uniformly bounded for all $|\theta| \leqq b$, all $k \in R$, and $0 \leqq n \leqq 2$

iv) $\left|f\left(\bar{E}_{0}\right)\right|^{2}+\left|f\left(-E_{0}\right)\right|^{2}>0$.

We remark that the class of functions satisfying condition $(\mathrm{R})$ is non-empty; for example, it contains $k^{2} \exp \left[-k^{2}\right]$. (In the language of the spin-boson Hamiltonian $[\mathrm{L}-\mathrm{Z}]$, this is the superohmic case.)

Extending (4.1) we define for $\theta$ real the unitary dilation operator $U(\theta)$ on $\mathscr{F}_{n}$ as follows (with abuse of notation we use $U(\theta)$ to denote the dilation operator on each $\left.\mathscr{F}_{n}\right)$ : for any $F$ in $\mathscr{F}_{n}(n \neq 0)$

$$
(U(\theta) F)\left(k_{1}, \ldots, k_{n}\right)=e^{-\frac{n \theta}{2}} F\left(e^{-\theta} k_{1}, \ldots, e^{-\theta} k_{n}\right) .
$$

Acting on $\mathscr{F}_{0}, U(\theta)$ is the identity. For real $\theta$ it follows that

$$
U(\theta) g_{n} U(\theta)^{-1}=e^{-\theta} g_{n}
$$

For real $\theta$ we define

$$
\begin{aligned}
g_{n}(\theta) & =U(\theta) g_{n} U(\theta)^{-1} \\
G_{n}(\theta) & =U(\theta) G_{n} U(\theta)^{-1}
\end{aligned}
$$

Both operators in (4.5) have an obvious analytic continuation, with the same domain as $g_{n}$, and we denote by $g_{n}(\theta), G_{n}(\theta)$ their continuations. The spectrum of $g_{n}(\theta)$ is $\left\{e^{-\theta} x: x \geqq 0\right\}$. Furthermore, an easy computation shows that for $\theta$ real, and $F \in \mathscr{F}_{n}$,

$$
\begin{aligned}
& P_{n+1} C(U(\theta) f) P_{n}^{*} F=U(\theta) P_{n+1} C(f) P_{n}^{*} U(\theta)^{-1} F, \\
& P_{n-1} A(U(\theta) f) P_{n}^{*} F=U(\theta) P_{n-1} A(f) P_{n}^{*} U(\theta)^{-1} F .
\end{aligned}
$$


Lemma 4.2. Assume that $f$ satisfies condition $(R)$, and let $F \in \mathscr{F}_{n}$ be in the domain of $g_{n}$. Then $P_{n+1} C\left(f_{\theta}\right) P_{n}^{*} F$ and $P_{n-1} A\left(f_{\bar{\theta}}\right) P_{n}^{*} F$ are the analytic continuations of (4.6) to the disk $|\theta| \leqq b$. Furthermore, for all $m \geqq \frac{1}{2}$,

$$
\begin{gathered}
\left\|g_{n+1}^{-m} P_{n+1} C\left(f_{\theta}\right) P_{n}^{*} F\right\| \leqq \sqrt{2}\left\|g_{1}^{-m} f_{\theta}\right\|\|F\| \\
\left\|P_{n-1} A\left(f_{\bar{\theta}}\right) P_{n}^{*} g_{n}^{-m} F\right\| \leqq\left\|g_{1}^{-m} f_{\bar{\theta}}\right\|\|F\| .
\end{gathered}
$$

Proof. The analyticity is proved in [OY], Proposition 2.5 , and we refer the reader there; property (ii) of condition ( $R$ ) is used in the proof. To derive (4.7), we modify the argument in Proposition 2.5 of [OY] as follows:

$$
\begin{aligned}
& \left(g_{n+1}^{-m} P_{n+1} C\left(f_{\theta}\right) P_{n}^{*} F\right)\left(k_{1}, \ldots, k_{n+1}\right) \\
& \quad=\frac{1}{\sqrt{n+1}}\left(\sum_{l=1}^{n+1}\left|k_{l}\right|\right)^{-m} \sum_{j=1}^{n+1} f_{\theta}\left(k_{j}\right) F\left(k_{1}, \ldots, \hat{k}_{j}, \ldots, k_{n+1}\right) .
\end{aligned}
$$

Therefore, we get

$$
\begin{aligned}
& \left|\left(g_{n+1}^{-m} P_{n+1} C\left(f_{\theta}\right) P_{n}^{*} F\right)\left(k_{1}, \ldots, k_{n+1}\right)\right|^{2} \leqq \frac{1}{n+1} \sum_{j=1}^{n+1} \frac{\left|f_{\theta}\left(k_{j}\right)\right|^{2}}{\left|k_{j}\right|^{2 m}}\left|F\left(k_{1}, \ldots, \hat{k}_{j}, \ldots, k_{n+1}\right)\right|^{2} \\
& \quad+\frac{1}{n+1} \sum_{i \neq j} \frac{\left|f_{\theta}\left(k_{i}\right)\right|\left|f_{\theta}\left(k_{j}\right)\right|}{\left(\sum_{l=1}^{n+1}\left|k_{l}\right|\right)^{2 m}}\left|F\left(k_{1}, \ldots, \hat{k}_{i}, \ldots, k_{n+1}\right)\right|\left|F\left(k_{1}, \ldots, \hat{k}_{j}, \ldots, k_{n+1}\right)\right| .
\end{aligned}
$$

Some elementary bounds show that

$$
\begin{aligned}
\left\|g_{n+1}^{-m} P_{n+1} C\left(f_{\theta}\right) P_{n}^{*} F\right\|^{2} \leqq & \left\|g_{1}^{-m} f_{\theta}\right\|^{2}\|F\|^{2} \\
& +n \int d k_{1} \ldots d k_{n+1} \frac{\left|f_{\theta}\left(k_{1}\right)\right|}{\left|k_{1}\right|^{m}} \frac{\left|k_{1}\right|^{1 / 2}\left|F\left(k_{1}, k_{3}, \ldots, k_{n+1}\right)\right|}{\left(\sum_{l \neq 2}\left|k_{l}\right|\right)^{1 / 2}} \\
& \cdot \frac{\left|f_{\theta}\left(k_{2}\right)\right|}{\left|k_{2}\right|^{m}} \frac{\left|k_{2}\right|^{1 / 2}\left|F\left(k_{2}, k_{3}, \ldots, k_{n+1}\right)\right|}{\left(\sum_{l \neq 1}\left|k_{l}\right|\right)^{1 / 2}} .
\end{aligned}
$$

Using Cauchy-Schwarz twice and the symmetry of $F$, this gives the stated result. The bound (4.8) is similar and simpler, and we omit the details. QED

We note that the complex conjugate appears in the analytic continuation $A\left(f_{\bar{\theta}}\right)$ because the operator is anti-linear.

The strategy of our proof of Theorem 3.2(a) is the following. Equation (2.16) gives an expression for $M_{0}(z)$ in terms of $M_{1}(z)$, and by iteration in terms of $M_{n}(z)$ for all $1 \leqq n \leqq N-1$. For real $\theta$, we can conjugate by the operator $U(\theta)$ and replace each $M_{n}(z)$ by $U(\theta) M_{n}(z) U(\theta)^{-1}$ for all $n \geqq 1$, without changing the value of $M_{0}(z)$ (since $U(\theta)$ is the identity on $\mathscr{F}_{0}$ ). Let us define for real $\theta$, and $n \geqq 0$,

$$
M_{n}(z, \theta)=U(\theta) M_{n}(z) U(\theta)^{-1} .
$$

We will prove that for $0 \leqq n \leqq N-1$, (4.9) can be analytically continued to the $\operatorname{disk}|\theta| \leqq b$, for $\beta$ sufficiently small, and for $z$ in the region $\operatorname{Im} z>1$, $\operatorname{Arg} z>\frac{\pi}{4}$. This will prove that $M_{0}(z, \theta)$ is analytic in $\theta$ and $z$ for all $|\theta| \leqq b$, and for all $z$ in the region indicated. Since it is independent of $\theta$ for real values, it is independent of $\theta$ for all $|\theta| \leqq b$. The next step will be to show that $M_{0}(z, i b)$ can 
be analytically continued to the region $\operatorname{Im} z>0,\left|\operatorname{Re} z-E_{0}\right| \leqq \alpha$ for some $\alpha>0$ (independent of $\beta$ ). Since $M_{0}(z)$ is analytic in this extended region, the equality $M_{0}(z)=M_{0}(z, i b)$ also holds there. We will derive uniform bounds on $M_{0}(z, i b)$ and its derivatives in this region; the same bounds hold for $M_{0}(z)$, and this proves Theorem 3.2(a).

Let us denote by $W$ the set of values $(\theta, z)$ satisfying $\operatorname{Im} z>1, \operatorname{Arg} z>\frac{\pi}{4}$ and $|\theta| \leqq b$.

Lemma 4.3. a) Let $(\theta, z) \in W$. Then for all $n \geqq 0$, and all $F \in \mathscr{F}_{n}$,

$$
\left\|\left(G_{n}(\theta)-z\right)^{-1} F\right\| \leqq 8\left\|\left(g_{n}+1\right)^{-1} F\right\| .
$$

b) $\left(G_{n}(\theta)-z\right)^{-1}$ is analytic in $W$.

Proof. a) Simple geometry shows that for $x \geqq 0$, and $(\theta, z) \in W$,

$$
\begin{aligned}
& \left|e^{-\theta} x-z\right| \geqq 2 \sqrt{2} \sin \frac{\pi}{16}, \\
& \left|e^{-\theta} x-z\right| \geqq \sin \frac{\pi}{8} e^{-\frac{\pi}{8} x},
\end{aligned}
$$

since $0<b<\frac{\pi}{8}$. Therefore

$$
\left|e^{-\theta} x-z\right| \geqq \frac{1}{8}(1+x) .
$$

This implies the stated result. Then (b) follows from (a) and the analyticity of the operator $\left(G_{n}(\theta)-z\right)$. QED

For $n \geqq 0$ and $|\theta| \leqq b$, we define by analogy with (2.8)

$$
\begin{aligned}
& C_{n+1}^{\theta}=Q_{n+1}^{*} P_{n+1} C\left(f_{\theta}\right) P_{n}^{*}, \\
& A_{n+1}^{\theta}=P_{n} A\left(f_{\bar{\theta}}\right) P_{n+1}^{*} Q_{n+1} .
\end{aligned}
$$

Since $Q_{n} Q_{n}^{*}=1$, Lemma 4.2 implies that

$$
\begin{aligned}
& \left\|g_{n+1}^{-1 / 2} Q_{n+1} C_{n+1}^{\theta}\right\| \leqq \sqrt{2}\left\|g_{1}^{-1 / 2} f_{\theta}\right\| \\
& \left\|A_{n+1}^{\theta} Q_{n+1}^{*} g_{n+1}^{-1 / 2}\right\| \leqq\left\|g_{1}^{-1 / 2} f_{\bar{\theta}}\right\|
\end{aligned}
$$

Proposition 4.4. There is $\beta_{4}>0$, independent of $N$, such that for all $|\beta| \leqq \beta_{4}$, and all $0 \leqq n \leqq N-1, M_{n}(z, \theta)$ is analytic in $W$.

Proof. We use (2.16) and an inductive argument. For all $n \leqq N,(2.16)$ implies that

$$
\begin{aligned}
M_{n-1}(z, \theta)= & A_{n}^{\theta} Q_{n}^{*}\left(G_{n}(\theta)-z-\beta^{2} M_{n}(z, \theta)\right)^{-1} Q_{n} C_{n}^{\theta} \\
= & A_{n}^{\theta} Q_{n}^{*}\left(G_{n}(\theta)-z\right)^{-1} Q_{n} C_{n}^{\theta} \\
& +\sum_{j=1}^{\infty} A_{n}^{\theta} Q_{n}^{*}\left(G_{n}(\theta)-z\right)^{-1}\left[\beta^{2} M_{n}(z, \theta)\left(G_{n}(\theta)-z\right)^{-1}\right]^{j} Q_{n} C_{n}^{\theta} .
\end{aligned}
$$


Lemmas 4.2 and 4.3 imply that for all $(\theta, z) \in W$,

$$
\begin{aligned}
\left\|M_{n-1}(z, \theta)\right\| \leqq & 8 \sqrt{2}\left\|g_{1}^{-1 / 2} f_{\theta}\right\|\left\|g_{1}^{-1 / 2} f_{\bar{\theta}}\right\| \\
& +\sqrt{2}\left\|g_{1}^{-1 / 2} f_{\theta}\right\|\left\|g_{1}^{-1 / 2} f_{\bar{\theta}}\right\|\left\|g_{n}^{1 / 2}\left(G_{n}(\theta)-z\right)^{-1}\right\| \\
& \cdot\left\|\left(G_{n}(\theta)-z\right)^{-1} g_{n}^{1 / 2}\right\| \sum_{j=1}^{\infty}\left[\beta^{2}\left\|M_{n}(z, \theta)\right\|\right]^{j} 8^{j-1} \\
\leqq & 8 \sqrt{2}\left\|g_{1}^{-1 / 2} f_{\theta}\right\|\left\|g_{1}^{-1 / 2} f_{\bar{\theta}}\right\|\left(1-\beta^{2}\left\|M_{n}(z, \theta)\right\|\right)^{-1}
\end{aligned}
$$

Now we make the inductive assumption that for some $n \leqq N$, the operator $M_{n}(z, \theta)$ is analytic in $W$, and satisfies the bound

$$
\left\|M_{n}(z, \theta)\right\| \leqq 16 \sqrt{2}\left\|g_{1}^{-1 / 2} f_{\theta}\right\|\left\|g_{1}^{-1 / 2} f_{\bar{\theta}}\right\| \text {. }
$$

We define $\beta_{4}$ as follows:

$$
\beta_{4}^{-2}=256 \sqrt{2} \sup _{|\theta| \leqq b}\left\{\left\|g_{1}^{-1 / 2} f_{\theta}\right\|\left\|g_{1}^{-1 / 2} f_{\bar{\theta}}\right\|\right\} .
$$

Then (4.11)-(4.14) imply that for $|\beta| \leqq \beta_{4}, M_{n-1}(z, \theta)$ is analytic in $W$, and satisfies the bound (4.13). Furthermore, since $M_{N}(z)=0$, analyticity and the bound (4.13) are trivially satisfied for $n=N$. Therefore they also hold for all $0 \leqq n \leqq$ $N-1$. QED

Proposition 4.4 implies that $M_{0}(z, \theta)$ is analytic in $W$, and hence independent of $\theta$, as discussed before. Therefore in particular,

$$
M_{0}(z)=M_{0}(z, i b) .
$$

Our next goal is to analytically continue $M_{0}(z, i b)$ to a vertical strip above the real axis. For any $\alpha>0$, with $\alpha<\frac{1}{2} E_{0}$, we define the region

$$
S_{\alpha}=\left\{z \in C|\operatorname{Im} z>0,| \operatorname{Re} z-E_{0} \mid \leqq \alpha\right\} .
$$

Simple geometry implies that for all $z \in S_{\alpha}$, and $x \geqq 0$,

$$
\begin{aligned}
\left|e^{-i b} x-z\right| & \geqq \frac{E_{0}}{2} \sin b, \\
\left|e^{-i b} x+E_{0}-z\right| & \geqq x \sin b .
\end{aligned}
$$

These bounds imply that for all $z \in S_{\alpha}$,

$$
\begin{aligned}
\left\|\left(g_{n}(i b)-z\right)^{-1}\right\| & \leqq \frac{2}{E_{0} \sin b}, \\
\left\|g_{n}(i b)\left(g_{n}(i b)+E_{0}-z\right)^{-1}\right\| & \leqq \frac{1}{\sin b} .
\end{aligned}
$$

The next result lies at the heart of this paper, and will be proved in Sect. 5 . For all $0 \leqq n \leqq N-1$ define

$$
\begin{aligned}
& L_{n}(z)=A_{n+1}^{i b} Q_{n+1}^{*}\left(G_{n+1}(i b)-z\right)^{-1} Q_{n+1} C_{n+1}^{i b}, \\
& N_{n}(z)=M_{n}(z, i b)-L_{n}(z) .
\end{aligned}
$$


Proposition 4.5. There are positive numbers $K_{5}, K_{6}$ and $\beta_{5}$, such that for all $|\beta| \leqq \beta_{5}$, all $z \in S_{\alpha}$, and all $n \geqq 0$,

i) $\left\|\left(g_{n}(i b)+E_{0}-z-\beta^{2} L_{n}(z)\right)^{-1}\right\| \leqq K_{5} \beta^{-2}$,

ii) $\left\|\left(g_{n}(i b)+E_{0}-z\right)\left(g_{n}(i b)+E_{0}-z-\beta^{2} L_{n}(z)\right)^{-1}\right\| \leqq K_{6}$,

iii) $\left\|\left(g_{n}(i b)+E_{0}-z-\beta^{2} L_{n}(z)\right)^{-1}\left(g_{n}(i b)+E_{0}-z\right)\right\| \leqq K_{6}$,

iv) $\left\|\left(g_{n}(i b)+E_{0}-z\right)^{1 / 2}\left(g_{n}(i b)+E_{0}-z-\beta^{2} L_{n}(z)\right)^{-1}\left(g_{n}(i b)+E_{0}-z\right)^{1 / 2}\right\| \leqq K_{6}$.

Proposition 4.5 will be proved in Sect. 5. We now use it to deduce Theorem 3.2(a). We will prove that $M_{0}(z, i b)$ has an analytic continuation to $S_{\alpha}$ for some $\alpha>0$, and at the same time derive uniform bounds on its norm and the norms of its first three derivatives in that region. Our proof follows the same lines as the proof of Proposition 4.4. We use (2.16) and an inductive argument to derive uniform bounds on $M_{n}(z, i b)$.

From (2.16) and (4.18) it follows that

$$
\begin{aligned}
N_{2 n}(z)= & \sum_{j=1}^{\infty} A_{2 n+1}^{i b} Q_{2 n+1}^{*}\left(g_{2 n+1}(i b)-z\right)^{-1} \\
& \cdot\left[\beta^{2} M_{2 n+1}(z, i b)\left(g_{2 n+1}(i b)-z\right)^{-1}\right]^{j} Q_{2 n+1} C_{2 n+1}^{i b} .
\end{aligned}
$$

Using the bound $\left\|g_{n}^{1 / 2}\left(g_{n}(i b)-z\right)^{-1}\right\| \leqq\left(\sqrt{2 / E_{0}}\right) / \sin b$, Lemma 4.2 and (4.19) imply that

$$
\left\|N_{2 n}(z)\right\| \leqq \frac{\sqrt{2}}{\sin b}\left\|g_{1}^{-1 / 2} f_{i b}\right\|\left\|g_{1}^{-1 / 2} f_{-i b}\right\| \sum_{j=1}^{\infty}\left[\beta^{2} \frac{2}{E_{0} \sin b}\left\|M_{2 n+1}(z, i b)\right\|\right]^{j} .
$$

We make the inductive assumption that for some $n$, satisfying $2 n+1 \leqq N$, the operator $M_{2 n+1}(z, i b)$ is analytic in $S_{\alpha}$, and satisfies the bound

$$
\left\|M_{2 n+1}(z, i b)\right\| \leqq \frac{2 \sqrt{2}}{\sin b} K_{6}\left\|g_{1}^{-1 / 2} f_{i b}\right\|\left\|g_{1}^{-1 / 2} f_{-i b}\right\| .
$$

Furthermore we define $\beta_{6}$ as follows:

$$
\beta_{6}^{-2}=\frac{4}{E_{0} \sin b} \frac{2 \sqrt{2}}{\sin b} K_{6}\left\|g_{1}^{-1 / 2} f_{i b}\right\|\left\|g_{1}^{-1 / 2} f_{-i b}\right\| .
$$

It follows that for $|\beta| \leqq \beta_{6}$ and $z \in S_{\alpha}$,

$$
\left\|N_{2 n}(z)\right\| \leqq \frac{4 \sqrt{2}}{E_{0} \sin ^{2} b}\left\|g_{1}^{-1 / 2} f_{i b}\right\|\left\|g_{1}^{-1 / 2} f_{-i b}\right\| \beta^{2}\left\|M_{2 n+1}(z, i b)\right\| .
$$

Now we return to (2.16), and deduce the following expansion:

$$
\begin{aligned}
M_{2 n-1}(z, i b)= & A_{2 n}^{i b} Q_{2 n}^{*}\left(G_{2 n}(i b)-z-\beta^{2} L_{2 n}(z)\right)^{-1} Q_{2 n} C_{2 n}^{i b} \\
& +\sum_{j=1}^{\infty} A_{2 n}^{i b} Q_{2 n}^{*}\left(G_{2 n}(i b)-z-\beta^{2} L_{2 n}(z)\right)^{-1} \\
& \cdot\left[\beta^{2} N_{2 n}(z)\left(G_{2 n}(i b)-z-\beta^{2} L_{2 n}(z)\right)^{-1}\right]^{j} Q_{2 n} C_{2 n}^{i b} .
\end{aligned}
$$

Proposition 4.5, combined with Lemma 4.2 (with $m=1 / 2$ and $m=1$ ), (4.17) and (4.23) imply that for all $z \in S_{\alpha}$, 


$$
\begin{aligned}
\left\|M_{2 n-1}(z, i b)\right\| \leqq & \frac{\sqrt{2}}{\sin b} K_{6}\left\|g_{1}^{-1 / 2} f_{-i b}\right\|\left\|g_{1}^{-1 / 2} f_{i b}\right\| \\
& +\frac{\sqrt{2} K_{6}^{2}}{\sin ^{2} b} \beta^{2}\left\|g_{1}^{-1} f_{-i b}\right\|\left\|g_{1}^{-1} f_{i b}\right\|\left\|N_{2 n}(z)\right\| \sum_{j=0}^{\infty}\left[K_{5}\left\|N_{2 n}(z)\right\|\right]^{j}
\end{aligned}
$$

Define

$$
\beta_{7}^{-2}=\max \left\{\beta_{6}^{-2}, \quad \frac{32 K_{5} K_{6}}{E_{0} \sin ^{3} b}\left\|g_{1}^{-1 / 2} f_{-i b}\right\|^{2}\left\|g_{1}^{-1 / 2} f_{i b}\right\|^{2}\right\} .
$$

Then for $z \in S_{\alpha}$ and $|\beta| \leqq \beta_{7}$, we have $K_{5}|| N_{2 n}(z)|| \leqq 1 / 2$, and so (4.24) implies

$$
\begin{aligned}
\left\|M_{2 n-1}(z, i b)\right\| \leqq & \frac{\sqrt{2} K_{6}}{\sin b}\left\|g_{1}^{-1 / 2} f_{-i b}\right\|\left\|g_{1}^{-1 / 2} f_{i b}\right\| \\
& +\frac{\sqrt{2} K_{6}^{2}}{\sin ^{2} b K_{5}} \beta^{2}\left\|g_{1}^{-1} f_{-i b}\right\|\left\|g_{1}^{-1} f_{i b}\right\| .
\end{aligned}
$$

Finally, define

$$
\beta_{5}^{-2}=\max \left\{\beta_{7}^{-2}, \frac{2 K_{6}\left\|g_{1}^{-1} f_{-i b}\right\|\left\|g_{1}^{-1} f_{i b}\right\|}{K_{5} \sin b\left\|g_{1}^{-1 / 2} f_{-i b}\right\|\left\|g_{1}^{-1 / 2} f_{i b}\right\|}\right\} .
$$

Then (4.26) implies that for $z \in S_{\alpha}$, and $|\beta| \leqq \beta_{5}, M_{2 n-1}(z, i b)$ satisfies the bound (4.21). If $N$ is odd, then (4.21) is automatically satisfied for $N=2 n+1$. If $N=2 n$ is even, then $N_{N-1}(z)=0$, and so (4.23) and (4.24) imply that

$$
\left\|M_{N-1}(z, i b)\right\| \leqq \frac{\sqrt{2} K_{6}}{\sin b}\left\|g_{1}^{-1 / 2} f_{-i b}\right\|\left\|g_{1}^{-1 / 2} f_{i b}\right\| .
$$

Therefore the bound (4.21) holds for all $n \geqq 0$, with $2 n+1 \leqq N$, and all $|\beta| \leqq$ $\beta_{5}$. The uniform convergence of the series (4.19) and (4.23) implies analyticity of $M_{2 n-1}(z, i b)$, for $z \in S_{\alpha}$.

In particular, we deduce that $M_{1}(z, i b)$ is analytic in $S_{\alpha}$. From (2.16) we have

$$
M_{0}(z, i b)=\sum_{j=0}^{\infty} A_{1}^{i b} Q_{1}^{*}\left(g_{1}(i b)-z\right)^{-1} \beta^{2 j}\left[M_{1}(z, i b)\left(g_{1}(i b)-z\right)^{-1}\right]^{j} Q_{1} C_{1}^{i b} .
$$

The bound (4.20) guarantees convergence of (4.28), and hence analyticity of $M_{0}(z, i b)$ in $S_{\alpha}$. The expression (4.28) also produces a uniform bound for $\left|M_{0}(z, i b)\right|$. Furthermore, for $\operatorname{Im} z \neq 0$,

$$
\begin{aligned}
M_{0}^{\prime}(z) & =A_{1}\left(h_{1}-z\right)^{-2} C_{1} \\
& =\sum_{j=0}^{\infty}(2 j+1) A_{1} Q_{1}^{*}\left(g_{1}-z\right)^{-2} \beta^{2 j}\left[M_{1}(z)\left(g_{1}-z\right)^{-1}\right]^{j} Q_{1} C_{1} .
\end{aligned}
$$

By repeating the analytic continuation arguments in this section, we deduce that $M_{0}^{\prime}(z, \theta)$ is analytic in $W$, and independent of $\theta$, for $|\beta| \leqq \beta_{5}$. The identity (4.29) and the bound (4.20) allows $M_{0}^{\prime}(z, i b)$ to be analytically continued to $S_{\alpha}$, where (4.29) implies that it is uniformly bounded, and equal to $M_{0}^{\prime}(z)$. A similar argument shows that higher derivatives of $M_{0}(z)$ are uniformly bounded in $S_{\alpha}$. This proves Theorem 3.2 (i). 
To prove Theorem 3.2 (ii) we must use property (iv) of Definition 4.1. Using (4.28), it follows that for $\operatorname{Im} z>0$,

$$
\begin{aligned}
M_{0}(z)=M_{0}(z, i b) & =A_{1}^{i b} Q_{1}^{*}\left(g_{1}(i b)-z\right)^{-1} Q_{1} C_{1}^{i b}+O\left(\beta^{2}\right) \\
& =A_{1} Q_{1}^{*}\left(g_{1}-z\right)^{-1} Q_{1} C_{1}+O\left(\beta^{2}\right) .
\end{aligned}
$$

Furthermore,

$$
\begin{aligned}
\lim _{\varepsilon \rightarrow 0+} A_{1} Q_{1}^{*}\left(g_{1}-E_{0}-i \varepsilon\right)^{-1} Q_{1} C_{1} & =\lim _{\varepsilon \rightarrow 0+} \int \frac{|f(p)|^{2}}{|p|-E_{0}-i \varepsilon} d p \\
& =\mathscr{P} \int \frac{|f(p)|^{2}}{|p|-E_{0}} d p+i \pi\left(\left|f\left(E_{0}\right)\right|^{2}+\left|f\left(-E_{0}\right)\right|^{2}\right)
\end{aligned}
$$

where ' $\mathscr{P}$ ' denotes the principal value. From these two results we deduce

$$
\lim _{\varepsilon \rightarrow 0+} \operatorname{Im} M_{0}\left(E_{0}+i \varepsilon\right)=\pi\left(\left|f\left(E_{0}\right)\right|^{2}+\left|f\left(-E_{0}\right)\right|^{2}\right)+O\left(\beta^{2}\right),
$$

which is positive for $\beta$ sufficiently small.

\section{Bounds on the Resolvent}

In this section we will prove Proposition 4.5. The proof relies on some properties of the following integral; for $\operatorname{Im} z>0$, define

$$
T(z)=\int_{-\infty}^{\infty} \frac{|f(p)|^{2}}{|p|-z} d p .
$$

From the assumption of dilatation analyticity for $f$, it follows that

$$
T(z)=\int_{-\infty}^{\infty} \frac{\overline{f_{-i b}(p)} f_{i b}(p)}{e^{-i b}|p|-z} d p .
$$

The estimate $\left|e^{-i b}\right| p|-z| \geqq|p| \sin b$ for $\operatorname{Im} z>0$ implies the bound

$$
|T(z)| \leqq \frac{1}{\sin b} \int_{-\infty}^{\infty} \frac{\left|f_{-i b}(p)\right|\left|f_{i b}(p)\right|}{|p|} d p .
$$

Let

$$
\tau=\sup _{\operatorname{Im} z>0}|T(z)|
$$

The interaction term $M_{n}(z)$ in the resolvent can be separated into two terms, which we will call the direct and exchange terms (these are defined below in (5.9) and (5.10)). The direct term yields the estimates we require; the exchange term causes a problem. However, the infrared problem comes from the behavior near momentum zero. In this regime, the contribution of the exchange term is very small, and goes to zero as the momentum goes to zero. The contribution of the direct term approaches a constant as the momentum goes to zero. Based on this observation, we introduce a splitting of the momentum space into two regions; in the large momentum region, there is no problem, and for small momenta the direct term dominates the exchange term. 
For ease of notation, let $\gamma(p)=|f(p)|^{2}+|f(-p)|^{2}$ for all $p \geqq 0$. Then for $\operatorname{Im} z>0$,

$$
T(z)=\int_{0}^{\infty} \frac{\gamma(p)}{p-z} d p .
$$

We define the constant $M$ which will appear in many of our bounds, as follows:

$$
M:=\max \left(\|\gamma\|_{2}, \sup _{p \geqq 0}\left\{\gamma(p),\left|\gamma^{\prime}(p)\right|,\left|\gamma^{\prime \prime}(p)\right|\right\}\right) .
$$

Lemma 5.1. Let $z=u+i v$, with $u>0, v>0$. Then

$$
|\operatorname{Im} T(u+i v)-\pi \gamma(u)| \leqq M v\left(u+\frac{3}{u}\right) .
$$

Proof. Note first that

$$
\begin{aligned}
\operatorname{Im} T(u+i v) & =v \int_{0}^{\infty} \frac{\gamma(p)}{(p-u)^{2}+v^{2}} d p \\
& =v \int_{0}^{2 u} \frac{\gamma(p)}{(p-u)^{2}+v^{2}} d p+v \int_{2 u}^{\infty} \frac{\gamma(p)}{(p-u)^{2}+v^{2}} d p
\end{aligned}
$$

Secondly,

$$
\pi \gamma(u)=v \int_{0}^{2 u} \frac{\gamma(u)}{(p-u)^{2}+v^{2}} d p+v \int_{|p-u| \geqq u} \frac{\gamma(u)}{(p-u)^{2}+v^{2}} d p
$$

The estimate $|\gamma(p)| \leqq M$ implies that

$$
\begin{aligned}
|\operatorname{Im} T(u+i v)-\pi \gamma(u)| & \leqq v\left|\int_{0}^{2 u} \frac{\gamma(p)-\gamma(u)}{(p-u)^{2}+v^{2}} d p\right|+3 v M \int_{2 u}^{\infty} \frac{1}{(p-u)^{2}} d p \\
& \leqq \frac{v}{2}\left|\int_{0}^{2 u} \frac{(p-u)^{2} \gamma^{\prime \prime}(s u+(1-s) p)}{(p-u)^{2}+v^{2}} d p\right|+3 v M \frac{1}{u}
\end{aligned}
$$

The estimate $\left|\gamma^{\prime \prime}(p)\right| \leqq M$ now gives

$$
|\operatorname{Im} T(u+i v)-\pi \gamma(u)| \leqq \frac{v}{2} M 2 u+3 v M \frac{1}{u} .
$$

QED

Lemma 5.2. There are positive numbers $\beta_{8}$ and $C_{1}$, such that for all $|\beta| \leqq \beta_{8}$, and $\operatorname{Im} z>0$,

$$
\left|E_{0}-z-\beta^{2} T(z)\right| \geqq \beta^{2} C_{1} .
$$

Proof. If $\left|E_{0}-z\right|>2 \beta^{2} \tau$, where $\tau$ is defined in (5.4), then

$$
\left|E_{0}-z-\beta^{2} T(z)\right| \geqq\left|E_{0}-z\right|-\beta^{2} \tau>\beta^{2} \tau .
$$


If $\left|E_{0}-z\right| \leqq 2 \beta^{2} \tau$, then $\operatorname{Im} z \leqq 2 \beta^{2} \tau$, and $\left|E_{0}-\operatorname{Re} z\right| \leqq 2 \beta^{2} \tau$. Therefore Lemma 5.1 implies that

$$
|\operatorname{Im} T(z)-\pi \gamma(\operatorname{Re} z)| \leqq M 2 \beta^{2} \tau\left(E_{0}+2 \beta^{2} \tau+\frac{3}{E_{0}-2 \beta^{2} \tau}\right) .
$$

By continuity of $\gamma(p)$ and our assumption that $\gamma\left(E_{0}\right) \neq 0$, there exists $r>0$, independent of $\beta, r<E_{0} / 2$, such that

$$
\inf _{\left|u-E_{0}\right|<r} \gamma(u) \geqq \frac{\gamma\left(E_{0}\right)}{2} .
$$

Then for $\beta^{2} \leqq r / 2 \tau$ and for $\left|E_{0}-z\right| \leqq 2 \beta^{2} \tau$, we have $\left|E_{0}-\operatorname{Re} z\right| \leqq r$, and so

$$
\begin{aligned}
|\operatorname{Im} T(z)| & \geqq \pi \gamma(\operatorname{Re} z)-M 2 \beta^{2} \tau\left(E_{0}+\frac{E_{0}}{2}+\frac{6}{E_{0}}\right) \\
& \geqq \frac{\pi}{2} \gamma\left(E_{0}\right)-2 \beta^{2} \tau M\left(\frac{3 E_{0}}{2}+\frac{6}{E_{0}}\right) .
\end{aligned}
$$

Let

$$
\beta_{8}^{2}=\min \left\{\frac{r}{2 \tau}, \frac{\frac{\pi}{4} \gamma\left(E_{0}\right)}{2 \tau M\left(\frac{3 E_{0}}{2}+\frac{6}{E_{0}}\right)}\right\} .
$$

Then for $|\beta| \leqq \beta_{8}$, and $\left|E_{0}-z\right| \leqq 2 \beta^{2} \tau,|\operatorname{Im} T(z)| \geqq \frac{\pi}{4} \gamma\left(E_{0}\right)$, and hence

$$
\begin{aligned}
\left|E_{0}-z-\beta^{2} T(z)\right| & \geqq \operatorname{Im} z+\beta^{2} \operatorname{Im} T(z) \\
& \geqq \frac{\pi}{4} \gamma\left(E_{0}\right) \beta^{2} .
\end{aligned}
$$

Taking $C_{1}=\min \left(\tau, \frac{\pi}{4} \gamma\left(E_{0}\right)\right)$ gives the desired result. QED

Lemma 5.3. There is a positive number $C_{2}$, such that for all $|\beta| \leqq \beta_{8}$, and $\operatorname{Im} z>0$,

$$
\left|E_{0}-z-\beta^{2} T(z)\right| \geqq C_{2}\left|E_{0}-z\right|
$$

Proof. From the proof of Lemma 5.2, if $\left|E_{0}-z\right|>2 \beta^{2} \tau$, then

$$
\left|E_{0}-z-\beta^{2} T(z)\right| \geqq\left|E_{0}-z\right|-\beta^{2} \tau>\frac{1}{2}\left|E_{0}-z\right| .
$$

If $\left|E_{0}-z\right| \leqq 2 \beta^{2} \tau$, then

$$
\left|E_{0}-z-\beta^{2} T(z)\right| \geqq \frac{\pi}{4} \gamma\left(E_{0}\right) \beta^{2} \geqq \frac{\frac{\pi}{4} \gamma\left(E_{0}\right)}{2 \tau}\left|E_{0}-z\right| .
$$

Taking $C_{2}=\min \left(\frac{1}{2}, \frac{\pi}{8 \tau} \gamma\left(E_{0}\right)\right)$ gives the desired result. QED

We now turn to the proof of Proposition 4.5. Recall that for all $0 \leqq n \leqq N-1$, $z \in S_{\alpha}$

$$
L_{n}(z)=A_{n+1}^{i b} Q_{n+1}^{*}\left(G_{n+1}(i b)-z\right)^{-1} Q_{n+1} C_{n+1}^{i b} .
$$


We also define for $0 \leqq n \leqq N-1, z \in S_{\alpha}$,

$$
\begin{aligned}
K_{n}(z) & =G_{n}(i b)-z, \\
J_{n}(z) & =K_{n}(z)-\beta^{2} L_{n}(z) .
\end{aligned}
$$

From now on we assume that $n$ is even, so $G_{n}(i b)=g_{n}(i b)+E_{0}$. It will be useful to separate (5.7) into the direct term $D_{n}(z)$ and the exchange term $E_{n}(z)$, so that $L_{n}(z)=D_{n}(z)+E_{n}(z)$. For $F \in \mathscr{F}_{n},($ recall $(5.1))$

$$
\begin{gathered}
\left(D_{n}(z) F\right)\left(k_{1}, \cdots, k_{n}\right)=T\left(z-e^{-i b}\left(\left|k_{1}\right|+\cdots+\left|k_{n}\right|\right)\right) F\left(k_{1}, \cdots, k_{n}\right), \\
\left(E_{n}(z) F\right)\left(k_{1}, \ldots, k_{n}\right)=\sum_{j=1}^{n} f_{i b}\left(k_{j}\right)\left\{\int \frac{\overline{f_{-i b}\left(k_{n+1}\right)} F\left(k_{1}, \ldots, \hat{k}_{j}, \ldots, k_{n+1}\right)}{e^{-i b}\left(\left|k_{1}\right|+\cdots+\left|k_{n+1}\right|\right)-z} d k_{n+1}\right\} .
\end{gathered}
$$

As a technical aid for the proof, we introduce a parameter $\delta>0$, which separates the low and high momentum regions. Its magnitude will be chosen later to satisfy some requirements. Having fixed $\delta$, we let $\Pi_{n}: \mathscr{F}_{n} \rightarrow \mathscr{F}_{n}$ denote the orthogonal projection onto the part $[0, \delta]$ of the spectrum of $g_{n}$, and let $\Omega_{n}=1-\Pi_{n}$. It will be convenient to use the following quantities:

$$
\begin{gathered}
\mu(p)=\sup _{z \in S_{\alpha}}\left|e^{-i b} p-z\right|^{-1}, \\
\rho(b)=\inf _{p \geqq \delta ; z \in S_{\alpha}}\left|e^{-i b} p+E_{0}-z\right| .
\end{gathered}
$$

Simple estimates show that (recall $\alpha<\frac{E_{0}}{2}$ )

$$
\mu(p) \leqq \frac{2}{\sin b}\left(p+\frac{E_{0}}{2}\right)^{-1} ; \rho(b) \geqq \delta \sin b .
$$

For any $F \in \mathscr{F}_{n}$,

$$
\begin{aligned}
\left\|J_{n}(z) F\right\|= & \left\|\Pi_{n} J_{n}(z) F+\Omega_{n} J_{n}(z) F\right\| \\
\geqq & \frac{1}{\sqrt{2}}\left\|\Pi_{n} J_{n}(z) F\right\|+\frac{1}{\sqrt{2}}\left\|\Omega_{n} J_{n}(z) F\right\| \\
\geqq & \frac{1}{\sqrt{2}}\left\{\left\|\Pi_{n} J_{n}(z) \Pi_{n} F\right\|+\left\|\Omega_{n} J_{n}(z) \Omega_{n} F\right\|\right\} \\
& -\frac{1}{\sqrt{2}}\left\{\left\|\Pi_{n} J_{n}(z) \Omega_{n} F\right\|+\left\|\Omega_{n} J_{n}(z) \Pi_{n} F\right\|\right\} .
\end{aligned}
$$

We will now obtain upper and lower bounds for the terms on the right side of (5.14). Since both $K_{n}(z)$ and $D_{n}(z)$ commute with $\Pi_{n}$ and $\Omega_{n}$, it follows that

$$
\Pi_{n} J_{n}(z) \Omega_{n}=-\beta^{2} \Pi_{n} E_{n}(z) \Omega_{n} .
$$

Furthermore, since the operator $\Pi_{n}$ restricts each momentum $\left|k_{j}\right|$ to the interval $[0, \delta],(5.10)$ and (5.13) imply 


$$
\begin{gathered}
\left|\left(\Pi_{n} E_{n}(z) \Omega_{n} F\right)\left(k_{1}, \ldots, k_{n}\right)\right| \leqq \frac{2}{\sin b} \sum_{j=1}^{n}\left|\Pi_{1} f_{i b}\left(k_{j}\right)\right| \\
\left\{\int\left|f_{-i b}\left(k_{n+1}\right)\right|\left(\left|k_{1}\right|+\cdots+\left|k_{n+1}\right|+\frac{E_{0}}{2}\right)^{-1}\left|\left(\Omega_{n} F\right)\left(k_{1}, \ldots, \hat{k}_{j}, \ldots, k_{n+1}\right)\right| d k_{n+1}\right\} .
\end{gathered}
$$

By adding on one extra term, this gives

$$
\left\|\Pi_{n} E_{n}(z) \Omega_{n} F\right\| \leqq \frac{2}{\sin b}\left\|P_{n} A\left(\left|f_{-i b}\right|\right)\left(g_{n+1}+\frac{E_{0}}{2}\right)^{-1} C\left(\left|\Pi_{1} f_{i b}\right|\right)\left(P_{n}^{*}\left|\Omega_{n} F\right|\right)\right\| .
$$

Using Lemma 4.2 (with $m=1 / 2$ ) and $\left\|\Omega_{n}\right\|=1$ produces the bound

$$
\left\|\Pi_{n} E_{n}(z) \Omega_{n} F\right\| \leqq \frac{2 \sqrt{2}}{\sin b}\left\|g_{1}^{-1 / 2} f_{-i b}\right\|\left\|\Pi_{1} g_{1}^{-1 / 2} f_{i b}\right\|\|F\|
$$

The "smallness" in (5.16) comes from the estimate

$$
|| \Pi_{1} g_{1}^{-1 / 2} f_{i b} \| \leqq \sqrt{2 \delta} \sup _{|k| \leqq \delta}\left\{|k|^{-1 / 2}\left|f_{i b}(k)\right|\right\} .
$$

From assumption (iii) of Definition 4.1, the last factor is finite. We define

$$
\sigma:=\sup _{|k| \leqq \delta ;|\theta| \leqq b}\left\{|k|^{-1 / 2}\left|f_{\theta}(k)\right|\right\}
$$

Then (5.16) implies the estimate

$$
\left\|\Pi_{n} E_{n}(z) \Omega_{n} F\right\| \leqq \sqrt{\delta} \sigma \frac{4}{\sin b}\left\|g_{1}^{-1 / 2} f_{-i b}\right\|\|F\| .
$$

A similar estimate shows that

$$
\left\|\Omega_{n} E_{n}(z) \Pi_{n} F\right\| \leqq \sqrt{\delta} \sigma \frac{4}{\sin b}\left\|g_{1}^{-1 / 2} f_{i b}\right\|\|F\|
$$

We now turn to the remaining two terms on the right side of (5.14). First,

$$
\Omega_{n} J_{n}(z) \Omega_{n} F=\Omega_{n} K_{n}(z) \Omega_{n} F-\beta^{2} \Omega_{n} L_{n}(z) \Omega_{n} F .
$$

The second term on the right side of (5.21) is bounded similarly to (4.19), (4.23) giving

$$
\left\|\Omega_{n} L_{n}(z) \Omega_{n} F\right\| \leqq \frac{\sqrt{2}}{\sin b}\left\|g_{1}^{-1 / 2} f_{i b}\right\|\left\|g_{1}^{-1 / 2} f_{-i b}\right\|\left\|\Omega_{n} F\right\|
$$

It is important to keep the projection operator in the last factor in (5.22). The first term on the right side of (5.21) is bounded from below by (recall (5.12))

$$
\left\|\Omega_{n} K_{n}(z) \Omega_{n} F\right\| \geqq \rho(b)\left\|\Omega_{n} F\right\| .
$$

We choose $\beta_{9}$ to satisfy

$$
\beta_{9}^{-2}=\frac{2 \sqrt{2}}{\sin b \rho(b)}\left\|g_{1}^{-1 / 2} f_{i b}\right\|\left\|g_{1}^{-1 / 2} f_{-i b}\right\|
$$


Then for all $|\beta| \leqq \beta_{9}$, and $z \in S_{\alpha}$, (5.21)-(5.24) and (5.13) imply

$$
\left\|\Omega_{n} J_{n}(z) \Omega_{n} F\right\| \geqq \frac{1}{2} \rho(b)\left\|\Omega_{n} F\right\| \geqq \frac{\delta \sin b}{2}\left\|\Omega_{n} F\right\| .
$$

The remaining term on the right side of (5.14) is given by

$$
\begin{aligned}
\Pi_{n} J_{n}(z) \Pi_{n} F= & \Pi_{n}\left[K_{n}(z)-\beta^{2} D_{n}(z)\right] \Pi_{n} F \\
& -\beta^{2} \Pi_{n} E_{n}(z) \Pi_{n} F .
\end{aligned}
$$

The last term on the right side of (5.26) is also bounded by (5.20) (multiplied by $\beta^{2}$ ). The first term on the right side of (5.26) is a multiplication operator. Let us write $x_{n}=\left|k_{1}\right|+\cdots+\left|k_{n}\right|$. Then for $F \in \mathscr{F}_{n}$ (recall (5.1))

$$
\begin{aligned}
\left(\left[K_{n}(z)-\beta^{2} D_{n}(z)\right] F\right)\left(k_{1}, \ldots, k_{n}\right)= & \left(e^{-i b} x_{n}+E_{0}-z-\beta^{2} T\left(z-e^{-i b} x_{n}\right)\right) \\
& \times F\left(k_{1}, \ldots, k_{n}\right) .
\end{aligned}
$$

The operator $\Pi_{n}$ imposes the restriction $x_{n} \leqq \delta$. Furthermore,

$$
\left|\operatorname{Im}\left(z-e^{-i b} x\right)\right|=\operatorname{Im} z+x \sin b>0,
$$

so Lemma 5.2 implies that for all $|\beta| \leqq \beta_{8}$,

$$
\left\|\left[K_{n}(z)-\beta^{2} D_{n}(z)\right] \Pi_{n} F\right\| \geqq \beta^{2} C_{1}\left\|\Pi_{n} F\right\| .
$$

Combining these estimates for (5.14), we find that for $\beta \leqq \min \left(\beta_{8}, \beta_{9}\right)$, and $z \in S_{\alpha}$

$$
\begin{aligned}
\left\|J_{n}(z) F\right\| \geqq & \frac{1}{\sqrt{2}} \beta^{2} C_{1}\left\|\Pi_{n} F\right\|+\frac{\delta \sin b}{2 \sqrt{2}}\left\|\Omega_{n} F\right\| \\
& -3 \sqrt{\delta} \beta^{2} \frac{4}{\sin b} \sigma\left\|g_{1}^{-1 / 2} f_{-i b}\right\|\|F\| .
\end{aligned}
$$

As our first condition on $\delta$ we require

$$
\delta \sin b \frac{1}{2 \sqrt{2}}>\frac{1}{\sqrt{2}} \beta^{2} C_{1} .
$$

Therefore (5.29) and the bound $\|F\| \leqq\left\|\Pi_{n} F\right\|+\left\|\Omega_{n} F\right\|$ give the estimate

$$
\left\|J_{n}(z)\right\| \geqq\left\{\frac{1}{\sqrt{2}} \beta^{2} C_{1}-3 \sqrt{\delta} \beta^{2} \frac{4}{\sin b}\left\|g_{1}^{-1 / 2} f_{i b}\right\| \sigma\right\}\|F\| .
$$

Our second condition on $\delta$ is that

$$
3 \sqrt{\delta} \frac{4}{\sin b}\left\|g_{1}^{-1 / 2} f_{i b}\right\| \sigma \leqq \frac{1}{2 \sqrt{2}} C_{1} .
$$

Combining (5.30) and (5.32) gives a condition that $\beta$ must satisfy, namely $|\beta| \leqq \beta_{10}$, where

$$
\beta_{10}^{2}=\frac{C_{1} \sin ^{3} b}{1152 \sigma^{2}\left\|g_{1}^{-1 / 2} f_{i b}\right\|^{2}} .
$$


With this condition, we deduce

$$
\left\|J_{n}(z) F\right\| \geqq \frac{C_{1} \beta^{2}}{2 \sqrt{2}}\|F\| .
$$

This proves Proposition 4.5(i), with $K_{5}=2 \sqrt{2} / C_{1}$. Part (ii) is proved in a similar way, by obtaining a lower bound on $\left\|J_{n}(z) K_{n}(z)^{-1} F\right\|$. Let us write $x_{n}=\left|k_{1}\right|+$ $\cdots+\left|k_{n}\right|$. First we note that

$$
J_{n}(z) K_{n}(z)^{-1} F=\left(1-\beta^{2} D_{n}(z) K_{n}(z)^{-1}\right) F-\beta^{2} E_{n}(z) K_{n}(z)^{-1} F .
$$

Furthermore,

$$
\begin{aligned}
& \left(E_{n}(z) K_{n}(z)^{-1} F\right)\left(k_{1}, \ldots, k_{n}\right) \\
& \quad=\sum_{j=1}^{n} f_{i b}\left(k_{j}\right)\left\{\int \frac{\overline{f_{-i b}\left(k_{n+1}\right)} F\left(k_{1}, \ldots, \hat{k}_{j}, \ldots, k_{n+1}\right)}{\left(e^{-i b} x_{n+1}-z\right)\left(e^{-i b}\left[x_{n+1}-k_{j}\right]+E_{0}-z\right)} d k_{n+1}\right\} .
\end{aligned}
$$

Recalling the estimate that for $\operatorname{Im} z>0,\left|e^{-i b} x+E_{0}-z\right| \geqq|x| \sin b$, we get

$$
\left|e^{-i b}\left[x_{n+1}-k_{j}\right]+E_{0}-z\right| \geqq\left|k_{n+1}\right| \sin b .
$$

We now repeat the argument leading to the bound (5.16), and this gives

$$
\left\|E_{n}(z) K_{n}(z)^{-1} F\right\| \leqq \frac{2 \sqrt{2}}{\sin ^{2} b}\left\|g_{1}^{-1 / 2} f_{i b}\right\|\left\|g_{1}^{-3 / 2} f_{-i b}\right\|\|F\| .
$$

Similar bounds hold for $\left\|K_{n}(z)^{-1} E_{n}(z) F\right\|$ and $\left\|K_{n}(z)^{-1 / 2} E_{n}(z) K_{n}(z)^{-1 / 2} F\right\|$. It remains to find a lower bound for $\left\|1-\beta^{2} D_{n}(z) K_{n}(z)^{-1}\right\|$. This is precisely the estimate given by Lemma 5.3, with $z$ there replaced by $z-e^{-i b} x_{n}$. Therefore we get the bound

$$
\left\|\left(1-\beta^{2} D_{n}(z) K_{n}(z)^{-1}\right) F\right\| \geqq C_{2}\|F\| \text {. }
$$

Let us define

$$
\beta_{11}^{2}=\frac{C_{2} \sin ^{2} b}{4 \sqrt{2}\left\|g_{1}^{-3 / 2} f_{-i b}\right\|\left\|g_{1}^{-1 / 2} f_{i b}\right\|} .
$$

Then for all $|\beta| \leqq \beta_{11},(5.37)$ implies Proposition 4.5 (ii) with $K_{6}=2 / C_{2}$. Since $D_{n}(z)$ and $K_{n}(z)$ commute, this proves (iii) and (iv) also.

\section{The Infinite Particle Number Limit}

In this final section we will prove that the amplitude $A(t)$ defined in (2.11) has a limit as $N \rightarrow \infty$, for all $t>0$. This will complete the proof of Theorem 3.1.

The idea of the proof is simple. We use the pointwise convergence of the resolvent $R(z)$ as $N \rightarrow \infty$; as we will see, it is sufficient to have pointwise convergence of $M_{0}(z)$. In order to apply this to the model, we deform the contour of integration in (2.13) as follows. Since $t>0$ and $|R(z)|$ is uniformly bounded (from (2.15)) for $|z|$ sufficiently large, we may deform the contour of integration in the lower half plane. Specifically, let $\Gamma$ be the following contour; the vertical halfline $\operatorname{Re} z=-2 E_{0}$, for $-\infty<\operatorname{Im} z \leqq 0$, followed by the semicircle $|z|=2 E_{0}$, for 
$\pi \geqq \operatorname{Arg} z \geqq 0$, followed by the line $\operatorname{Arg}\left(z-2 E_{0}\right)=-b / 2$, for $0 \geqq \operatorname{Im} z>-\infty$. We shall prove the following result.

Proposition 6.1. There are constants $\beta_{12}, C_{3}$, independent of $N$, such that $|R(z)| \leqq C_{3}$ for all $|\beta| \leqq \beta_{12}$, and all $z \in \Gamma$. Furthermore, $\lim _{N \rightarrow \infty} R(z)$ exists for all $z \in \Gamma$.

We will first use Proposition 6.1 to prove Theorem 3.1 (b). For $t>0$, we may deform the contour of integration in (2.13) to get

$$
A(t)=\frac{1}{2 \pi i} \int_{\Gamma} e^{-i z t} R(z) d z .
$$

For all $t>0$, Proposition 6.1 implies that for all $z \in \Gamma$,

$$
\left|e^{-i z t} R(z)\right| \leqq C_{3} \exp (t \operatorname{Im} z)
$$

The function $\exp (t \operatorname{Im} z)$ is integrable on $\Gamma$ for $t>0$. Therefore Proposition 6.1 and dominated convergence imply that

$$
\lim _{N \rightarrow \infty} A(t)=\frac{1}{2 \pi i} \int_{\Gamma} e^{-i z t} \lim _{N \rightarrow \infty} R(z) d z
$$

which proves Theorem 3.1(a).

Proof of Proposition 6.1. The proof uses the analytic continuation of $M_{0}(z)$, as in Sect. 4. In this case the continuation is considerably simpler, since we avoid the regions near 0 and $E_{0}$. First recall from Sect. 4 that $M_{0}(z, \theta)$ is analytic in the region $W$, which implies that $M_{0}(z)=M_{0}(z, i b)$ in $W$.

The complement of the contour $\Gamma$ has two connected components. Let $\tilde{\Gamma}$ denote the closure of that component which does not contain zero. Then simple geometry shows that for all $z \in \tilde{\Gamma}$, the following estimates hold: for all $x \geqq 0$,

$$
\begin{aligned}
\left|e^{-i b} x-z\right| & \geqq \max \left\{2 E_{0}, 2 E_{0} \sin b, x \cos b, x \sin (b / 2)\right\}, \\
\left|e^{-i b} x+E_{0}-z\right| & \geqq \max \left\{3 E_{0}, E_{0} \sin b, x \cos b, x \sin (b / 2)\right\} .
\end{aligned}
$$

The estimates (6.4) imply that for all $z \in \tilde{\Gamma}$, and all $n \geqq 0$, all $F \in \mathscr{F}_{n}$,

$$
\left\|\left(G_{n}(i b)-z\right)^{-1} F\right\| \leqq \frac{2}{\sin (b / 2)}\left\|\left(g_{n}+E_{0}\right)^{-1} F\right\| .
$$

The estimate (6.5) allows us to analytically continue each operator $M_{n}(z, i b)$ to $\tilde{\Gamma}$. The identity (2.16) gives

$$
\begin{aligned}
M_{n}(z, i b)= & A_{n+1}^{i b} Q_{n+1}^{*}\left(G_{n}(i b)-z\right)^{-1} Q_{n+1} C_{n+1}^{i b} \\
& +\sum_{j=1}^{\infty} A_{n+1}^{i b} Q_{n+1}^{*}\left(G_{n}(i b)-z\right)^{-1} \\
& \cdot\left[\beta^{2} M_{n+1}(z, i b)\left(G_{n+1}(i b)-z\right)^{-1}\right]^{j} Q_{n+1} C_{n+1}^{i b} .
\end{aligned}
$$

The usual inductive argument can be used now. Indeed, (6.5) implies that 


$$
\begin{aligned}
\left\|M_{n}(z, i b)\right\| \leqq & \frac{2}{\sin (b / 2)} \sqrt{2}\left\|g_{1}^{-1 / 2} f_{i b}\right\|\left\|g_{1}^{-1 / 2} f_{-i b}\right\| \\
& +\sum_{j=1}^{\infty} \frac{2}{\sin (b / 2)} \sqrt{2 / E_{0}}\left\|g_{1}^{-1 / 2} f_{-i b}\right\| \\
& \cdot\left[\beta^{2}\left\|M_{n+1}(z, i b)\right\|\right]^{j}\left(\frac{2}{E_{0} \sin (b / 2)}\right)^{j-1} \frac{2}{\sin (b / 2)} \frac{1}{\sqrt{E_{0}}}\left\|g_{1}^{-1 / 2} f_{i b}\right\| .
\end{aligned}
$$

The series in (6.7) converges provided that $2 \beta^{2}\left\|M_{n+1}(z, i b)\right\| \leqq E_{0} \sin (b / 2)$. By imitating the argument in the proof of Proposition 4.4, and using the starting condition $M_{N}(z)=0$, we deduce analyticity of $M_{n}(z, i b)$ in $\tilde{\Gamma}$ for all $N \geqq n \geqq 0$. Comparison with that proof also shows that for $\beta^{2}$ sufficiently small, we have the bound

$$
\left\|M_{n}(z, i b)\right\| \leqq \frac{4 \sqrt{2}}{\sin (b / 2)}\left\|g_{1}^{-1 / 2} f_{i b}\right\|\left\|g_{1}^{-1 / 2} f_{-i b}\right\|
$$

The bound (6.8) in the case $n=0$ implies that for $\beta^{2}$ sufficiently small, the function $R(z)=\left(E_{0}-z-\beta^{2} M_{0}(z)\right)^{-1}$ is also uniformly bounded in the region $\tilde{\Gamma}$. This proves the first statement in Proposition 6.1.

To derive the existence of the limit $N \rightarrow \infty$ of $R(z)$, it is sufficient to prove the existence of $\lim _{N \rightarrow \infty} M_{0}(z)$. We do this by showing that $\left\{M_{0}(z)\right\}$ is a Cauchy sequence in $N$.

Let $\tilde{N} \geqq N$ be any integer, and let $\left\{\tilde{M}_{n}(z, i b)\right\}$ denote the operators defined in (2.14), with the cutoff $N$ replaced by $\tilde{N}$. Then the above estimates show that $\left\|\tilde{M}_{N}(z, i b)\right\|$ satisfies the bound $(6.8)$, while $M_{N}(z)=0$, of course. For any $n \leqq N$, let

$$
a_{n}=\sup _{z \in \tilde{\Gamma}}\left\|\tilde{M}_{n}(z, i b)-M_{n}(z, i b)\right\| \text {. }
$$

From (2.16) and (6.6) we deduce that

$$
\begin{aligned}
\tilde{M}_{n}(z, i b)-M_{n}(z, i b)= & A_{n+1}^{i b} Q_{n+1}^{*}\left(G_{n+1}(i b)-z-\beta^{2} M_{n+1}(z, i b)\right)^{-1} \\
& \cdot\left[\beta^{2}\left(M_{n+1}(z, i b)-\tilde{M}_{n+1}(z, i b)\right)\right] \\
& \cdot\left(G_{n+1}(i b)-z-\beta^{2} \tilde{M}_{n+1}(z, i b)\right)^{-1} Q_{n+1} C_{n+1}^{i b} .
\end{aligned}
$$

Also (6.5) and (6.8) imply that for $\beta^{2}$ sufficiently small, we have the following bound:

$$
\left\|\left(G_{n}(i b)-z-\beta^{2} M_{n}(z, i b)\right)^{-1} F\right\| \leqq \frac{2}{\sin (b / 2)}\left\|\left(g_{n}+\frac{E_{0}}{2}\right)^{-1} F\right\| .
$$

Applying this to (6.10) gives

$$
a_{n} \leqq \sqrt{2} \beta^{2}\left(\frac{2}{\sin (b / 2)}\right)^{2}\left\|g_{1}^{-1} f_{i b}\right\|\left\|g_{1}^{-1} f_{-i b}\right\| a_{n+1}
$$

Therefore for $\beta^{2}$ sufficiently small, there is a constant $c>0$ such that for all $n \leqq N$,

$$
a_{n} \leqq c \beta^{2} a_{n+1} .
$$


From (6.8) we conclude that for some constant $c_{1}$,

$$
a_{0} \leqq\left(c \beta^{2}\right)^{N} c_{1}
$$

Therefore for $\beta^{2}$ sufficiently small, and for all $z \in \tilde{\Gamma}$,

$$
\lim _{N, \tilde{N} \rightarrow \infty}\left|\tilde{M}_{0}(z)-M_{0}(z)\right|=0 .
$$

Acknowledgements. The author thanks L. Gross for many useful discussions on resonances and radiation.

\section{References}

[AC] Aguilar, J., Combes, J.M.: A class of analytic perturbations for one-body Schrodinger Hamiltonians. Commun. Math. Phys. 22, 269-279 (1971)

[BC] Balslev, E., Combes, J.M.: Spectral properties of many-body Schrodinger operators with dilatation-analytic interactions. Commun. Math. Phys. 22, 280-294 (1971)

[Bl] Blanchard, Ph.: Discussion mathématique du modèle de Pauli et Fierz relatif à la catastrophe infrarouge. Commun. Math. Phys. 15, 156-172 (1969)

[BN] Bloch, F., Nordsieck, A.: Note on the radiation field of the electron. Phys. Rev. 52, 54-59 (1937)

[CMR] Combe, Ph., Mourre, E., Richard, J-L.: Photoionisation of atoms and Möller operators. Commun. Math. Phys. 43, 161-170 (1975)

[Da] Davies, E.B.: Dynamics of a multilevel Wigner-Weisskopf atom. J. Math. Phys. 15, 20362041 (1974)

[Di] Dirac, P.A.M.: The Principles of Quantum Mechanics. Oxford: Oxford University Press, 4th ed. 1978

[DE] Dittrich, J., Exner, P.: A non-relativistic model of two-particle decay I. Galilean invariance. Czech. J. Phys. B37, 503-515 (1987); II. Reduced resolvent, Czech. J. Phys. B37, 10281034 (1987); III. The pole approximation, Czech. J. Phys. B38, 591-610 (1988); IV. Relation to the scattering theory, spectral concentration, and bound states, Czech. J. Phys. B39, 121-138 (1989)

[EY] Eberly, J.H., Yoo, H.: Dynamical theory of an atom with two or three levels interacting with quantized cavity fields. Phys. Rep. 118, 239 (1985)

[F] Friedrichs, K.O.: On the perturbation of continuous spectrum. Commun. Pure Applied Math. 1, no. 4, 361-406 (1948)

[Froh] Fröhlich, J.: On the infrared problem in a model of scalar electrons and massless, scalar bosons. Ann. Inst. Henri Poincare A19, 1-103 (1974)

[FrMS] Fröhlich, J., Morchio, G., Strocchi, F.: Infrared problem and spontaneous breaking of the Lorentz group in QED. Phys. Lett. 89B, 61-64 (1979)

[G] Gross, L.: The relativistic polaron without cutoffs. Commun. Math. Phys. 31, 25-73 (1973)

[He] Heitler, W.: The Quantum Theory of Radiation. London: Oxford University Press, third edition, 1954

[Hu] Hunziker, W.: Resonances, metastable states, and exponential decay laws in perturbation theory. Commun. Math. Phys. 132, 177-188 (1990)

[Ki] King, C.: Exponential decay near resonance, without analyticity. Lett. Math. Phys. 23, 215-222 (1991)

[L-Z] Leggett, A.J., Chakravarty, S., Dorsey, A.T., Fisher, M., Garg, A., Zwerger, W.: Dynamics of the dissipative two-state system. Rev. Mod. Phys. 59, 1 (1987)

[N1] Nelson, E.: Analytic vectors. Ann. Math. 70, 572-615 (1959)

[N2] Nelson, E.: Interaction of non-relativistic particles with a quantized scalar field. J. Math. Phys. 5, 1190-1197 (1964) 
[OY] Okamoto, T., Yajima, K.: Complex Scaling Technique in non relativistic QED, Ann. Inst. Henri Poincaré, 42, no. 3, 311-327 (1985)

[S1] Simon, B.: Resonances in N-body quantum systems with dilatation analytic potentials and the foundations of time-dependent perturbation theory. Ann. Math. 97, 247-274 (1973)

[S2] Simon, B.: Resonances and complex scaling: A rigorous overview. Int. J. Quant. Chem. 14, 529-542 (1978)

[Sk] Skibsted, E.: Truncated Gamow functions, $\alpha$-decay and the exponential law. Commun. Math. Phys. 104, 591-604 (1986)

[SD] Spohn, H., Dümcke, R.: Quantum tunneling with dissipation and the Ising model over R. J. Stat. Phys. 41, 389-423 (1985)

[St] Stenholm, S.: Quantum theory of electromagnetic fields interacting with atoms and molecules. Phys. Rep. 6C, 1 (1973)

Communicated by B. Simon 